\title{
Effect of zinc deficiency on cognitive function in females with iron deficiency anemia
}

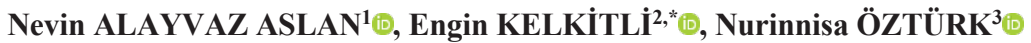 \\ ${ }^{1}$ Department of Hematology, Faculty of Medicine, Pamukkale University, Denizli, Turkey \\ ${ }^{2}$ Department of Hematology, Faculty of Medicine, Ondokuz Mayıs University, Samsun Turkey \\ ${ }^{3}$ Department of Biochemistry, Faculty of Medicine, Atatürk University, Erzurum, Turkey
}

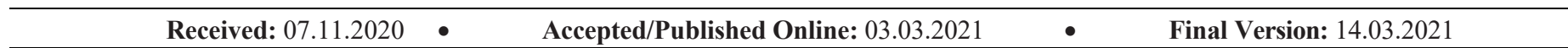

Abstract

Iron deficiency and zinc deficiency are known to disturb cognitive functions especially in infants and preschool children. Our purpose was to evaluate the effect of zinc deficiency and iron deficiency on cognitive function (with Montreal Cognitive Assessment [MoCA] score) in females. We designed a randomized controlled prospective study. 41 iron deficient female patients and 40 healthy volunteers were involved in the study. The average MoCA score \pm SD was 24,63 \pm 4,3 (range 11-30) in iron deficiency anemia (IDA), and 27.43 \pm 2.02 (23-30) in healthy volunteers. Zinc level was $97.06(40.8-146.8) \mathrm{mg} / \mathrm{dl}$ and 257.5 (128.2-463.7) in IDA and healthy volunteers respectively. MoCA score ( $\mathrm{r}=0.803, \mathrm{p}<0,01)$, were significantly correlated with Zinc level in iron deficient group. We found that there was a statistically important linear relationship between MoCA score and zinc level in female patients with iron deficiency anemia.
\end{abstract}

Keywords: anemia, zinc, cognitive dysfunction, iron deficiency

\section{Introduction}

Cognitive impairments are major intimidation to functional independence, quality of life, and long-term health and survival. Cognitive impairments are common in patients with anemia. Anemic patients have a higher risk of cognitive impairment compared with non-anemic individuals (Wang et al., 2006). One of the most common nutritional problems in the world is iron deficiency anemia and it is associated with serious health risks including abnormal motor and mental development in infancy. In cases of severe anemia, there is increased maternal and infant mortality risk and also there is increased risk of premature birth (Fang et al., 2011, Hershko and Camaschella, 2014). Some observational and retrospective studies have showed that iron deficiency is also related to impaired cognitive function. Iron deficiency disturbs the neurometabolism, neurotransmitters and myelination (Lozoff and Georgieff, 2006, Abdelhamid et al., 2010). Zinc, is an essential trace element for growth and development of humans. Zinc is also closely related to cognitive function. Because it is an important essential element for neurogenesis and synaptogenesis. Zinc deficiency can affect cognitive development by alterations in attention, activity, motor development and neuropsychological behavior (Bhatnagar and Taneja, 2001). The Montreal Cognitive Assessment (MoCA) is a new international brief cognitive screening tool which was developed for the detection of mild cognitive impairment (Phillips et al., 2005). Recent studies showed that the MoCA was more sensible to mild cognitive impairment than the MiniMental State Examination (MMSE) (Churilov et al., 2013). MoCA score has been validated to Turkish verison in 2010 by Cangöz et al (Cangöz et al., 2010).

Notwithstanding widespread use, there have been few investigations in the literature about the relationship between the Montreal Cognitive Assessment (MoCA) score and the iron deficiency anemia with or without zinc deficiency. There are some investigations those show the relationship between cognitive impairments and iron deficiency or zinc deficiency separately, especially in children and adolescent. The aim of this study is to evaluate cognitive function of female patients with iron deficiency anemia (IDA) by using MoCA score and to investigate effect of zinc deficiency on cognitive function in patients with IDA. 


\section{Material and method}

Between January 2014 and January 2015, we conducted study with 41 female patients with iron deficiency anemia who were admitted to hematology department of Erzurum Research Hospital and 40 age and gender-matched healthy control subjects. Approval for the study was granted by the Local Ethics Committee. All patients were asked to provide a detailed history and were subjected to a physical examination. A full blood count was performed on an automated analyzer within $60 \mathrm{~min}$ of collection by automated counter (Sysmex XE2100, SYSMEX Corporation, Kobe) for hemogram parameters. The Study complies with ethical standards by the given permission from Local Ethics Committee at 19 November 2013 with a number 12. The study was designed and conducted per the ethical principles of the Declaration of Helsinki. The informed consents were obtained from all patients and control group before the enrollment to the study

Peripheral blood smear analysis to define the anemia type, as well as all other laboratory tests, were conducted in the department of hematology. Those with microcytic indices $(\mathrm{MCV}<80 \mathrm{fL})$ and microcytic hypochromic picture on peripheral smear and hypochromic index $(\mathrm{MCH}<26 \mathrm{pg} / \mathrm{cell})$ were considered to have iron deficiency anemia, which was then confirmed by low serum ferritin levels $(<10 \mathrm{ng} / \mathrm{mL})$. Serum ferritin levels were measured using the chemiluminescence technique on Advia Centaur XP (Siemens Healthcare Diagnostics, Germany). All the laboratory parameters analyzed for the patients were also analyzed for the control group.

Blood specimen taken from patients and healthy adults were put into hemogram tubes and gel-containing biochemistry tubes. The biochemistry tubes were centrifuged at $3500 \mathrm{rpm}$ for $10 \mathrm{~min}$, and the serum samples were stored at $-80^{\circ} \mathrm{C}$ until the day of analysis. Serum zinc levels were measured in the flame unit of an atomic absorption spectrophotometer (Perkin Elmer Analyst 800 model, AAS, USA). Serum levels of zinc were calculated using standard graphics created for each element. Serum zinc level below the $100 \mathrm{mcg} / \mathrm{dl}$ was considered as zinc deficiency.

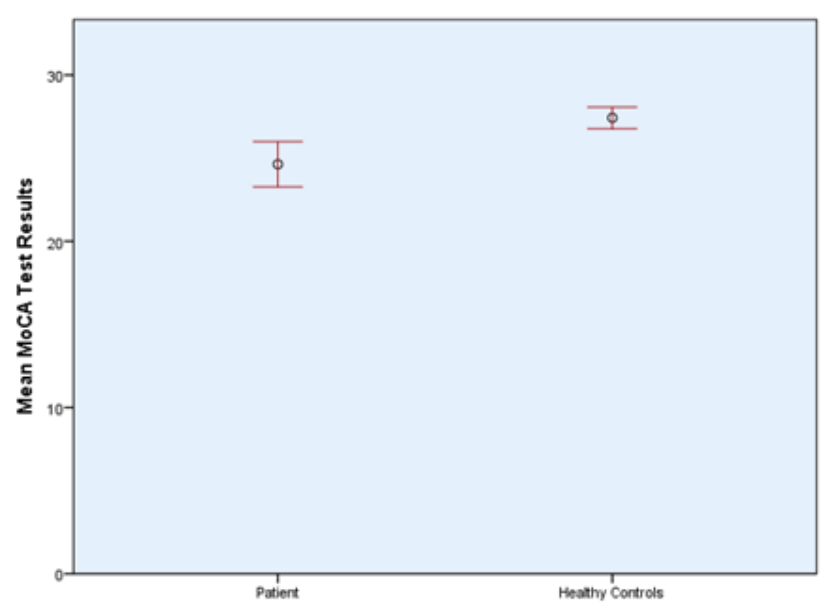

Fig. 1. MoCA score in anemic patients and control groups
Patients were screened for cognitive impairment using the Turkish version of MoCA test (https://www.mocatest.org/pdf_files/test/MOCA-Test-

Turkish.pdf). MoCA score has been validated to Turkish verison in 2010 by Cangöz et al. (Cangöz et al., 2010). Eight cognitive domain scores and the MoCA total score were used in this secondary analysis. MoCA has a total scoring range of 0 to 30 . A score below 17 is considered dementia, 17 to 21 is considered moderate cognitive impairment, 22 to 25 is considered mild cognitive impairment and score of 26 to 30 is considered normal. Validated use of the MoCA is based on explanation of the total score, with a score of 26 or less indicative of cognitive impairment.

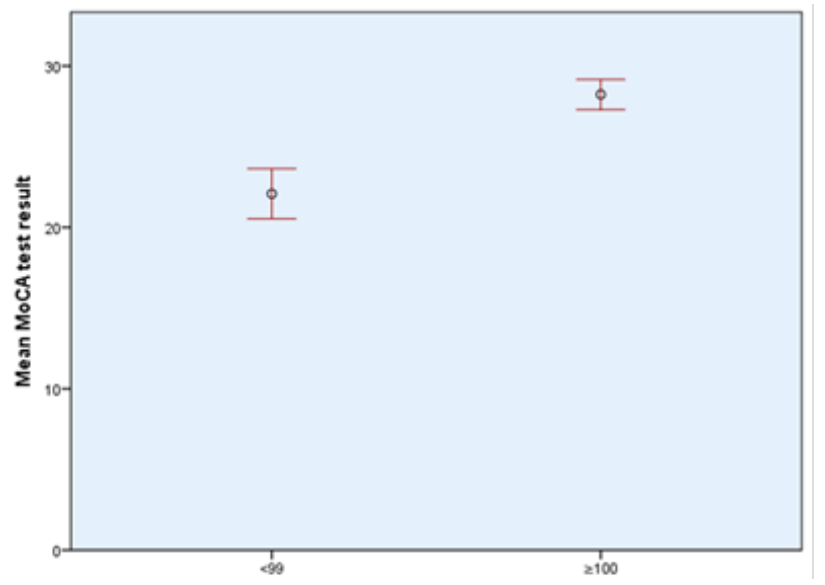

Fig. 2. MoCA score in iron deficient females with normal and low zinc levels

Patients with alcoholism, intestinal malabsorption syndromes, chronic renal disease, dimorphic anemia, extensive burns, malignancy, hypothyroidism, exfoliative dermatitis, HIV infection, pregnancy or lactation, connective tissue disease, neurocognitive problems (confusion, transitional ischemic attack, short-term memory loss, cerebral vascular accident, delirium, or dementia) documented in the medical history; and inability to answer questionnaires independently due to visual acuity or language barriers and active infection were excluded from the study. Patients who had been treated with parenteral or oral iron in the previous 3 months and those who were smokers were not included in the study.

Statistical analyses were performed using the statistical software program, SPSS, for Windows version 21.0 (SPSS; Chicago, IL, USA). Results were given as mean \pm standard deviation (SD). Since variables showed normal distribution with the Kolmogorov-Smirnov test, a parametric independent $t$ test was performed to detect statistical differences between the two groups. Pearson's correlations were used for correlation analyses. A value of $\mathrm{p}<0.05$ was considered statistically significant

\section{Results}

Table 1 shows the demographic characteristics of the patients. The mean age of patients with iron deficiency anemia was 
$33.1 \pm 12.5$ years (range, $16-65$ years) and the mean age of the healthy control subjects was 34.7 \pm 5.6 years (range, 21-45 years). A statistically important difference was determined between the two groups in the MoCA test score. MoCA score were lower in anemic patients $(24.63 \pm 4.3$ [range 11-30]) than in the control subjects $(27.43 \pm 2.02$ [range 23-31]), $(\mathrm{p}<0.001)$. The MoCA test score in the anemic patients and control groups are shown in Figure 1. Serum levels of zinc were all normal in healthy controls so we couldn't compare MoCA score and zinc level in whole study group. When we analyzed MoCA score in iron deficient patients, we showed that zinc deficient IDA patients had statistically significant low MoCA score (mean MoCA score: $22.08 \pm 3.68,95 \% \mathrm{CI}$ 13-97-26.27) compared to IDA patient with normal zinc level (mean MoCA score: 28.24 \pm 1.82 , 95\% CI 20.32-32.62) $(\mathrm{p}<0.05)$ (Figure 2). There was an important linear relationship between zinc level and MoCA score $(\mathrm{r}=0.803$; $\mathrm{p}<0.01$ ) in iron deficient females (Table 2).

Table 1. Characteristics of the participants, zinc level and MoCA

\begin{tabular}{|c|c|c|}
\hline & $\begin{array}{c}\text { Patients with Iron } \\
\text { Deficiency Anemia }\end{array}$ & Healthy controls \\
\hline Age (years) & $33.1 \pm 12.5$ & $34.7 \pm 5.6$ \\
\hline MoCA Score & $24.63 \pm 4.3(11-30)$ & $27.43 \pm 2.02(23-30)$ \\
\hline \multirow{2}{*}{ Zinc (mg/dL) } & $97.05 \pm 25.4$ & $257.05 \pm 91.4$ \\
& $(40.8-146.8)$ & $(128.2-463.7)$ \\
\hline
\end{tabular}

Table 2. Relationship between zinc levels in iron deficient patients and MoCA scores

\begin{tabular}{|c|c|c|c|}
\hline & \multicolumn{2}{|c|}{ Zinc Levels (mg/dl) } & Total \\
\hline & $<99$ & $\geq 100$ & \\
\hline MoCA Score & & & $21(51 \%)$ \\
$<25$ & $20(87 \%)$ & $1(5.5 \%)$ & $20(49 \%)$ \\
$>26$ & $3(13 \%)$ & $17(94.5 \%)$ & \\
\hline Total & $23(56 \%)$ & $18(44 \%)$ & $41(100 \%)$ \\
\hline
\end{tabular}

Our study showed that the MoCA score was lower in anemic patients $(24.67 \pm 4.24)$ than healthy controls $(27.42 \pm 2.02) ; \mathrm{p}<0.001$. The results of this study also determined the relationship between zinc deficiency and MoCA score. Important point in this study is; zinc deficient subjects had lower MoCA (22.08) score independent from their iron deficiency status. In iron deficient patient group $87 \%$ of patients with low zinc level had also low Moca score whereas only $5.5 \%$ of patients with normal zinc levels had low Moca score and it is statistically significant $(\mathrm{p}<0.05)$.

Zinc is a well-known element for neurocognitive development by contributing structure of several enzymes such as RNA and DNA polymerases and histone deacetylase which are needed for DNA replication and cellular proliferation (Aruga, 2004). Also zinc transporter genes are crucial for neurodevelopment and Zinc family of genes controls the process of neurulation (Aruga, 2004, Chowanadisai et al., 2013, Pfaender et al., 2016). Zinc deficiency leads to cognitive and emotional deficits that might be associated with neurodevelopmental disorders in animal models (Grabrucker et al., 2016).
A study from China showed that low zinc level was significantly associated with higher total behavior problems in preschoolers but not with low iron status. Also, both iron and zinc deficiency positively correlate with behavior problems of the preschool children. They concluded that zinc supplementation may positively affect cognitive functions in middle aged and older adults (Maylor et al., 2006).

In our study we showed that anemia had close relation with cognitive impairment but independent of iron deficiency anemia, zinc deficiency affected cognitive function negatively and caused low MoCA score in females. These findings are important when considering the patients age group. Because we didn't investigate pediatric patients or geriatric patients and our patient group was composed of mostly middle aged and young adult females.

Another study investigates the effects of zinc deficiency to cognitive functions in older residents of nursing homes by using Abbreviated Mental Test Score (AMTS) (MarkiewiczZukowska et al., 2015). It indicates that serum zinc level correlated with mental efficiency and was statistically significantly lower in older people with depression and mental impairment.

Limitation of our study is having only female patient and control group and also small sample size. So, we couldn't generalize our result to whole adult iron deficient patients. Because our cohort consisted only of females, emotional and cognitive changes that occur during menstrual period may have affected the MoCA scores. Randomized multicenter studies with larger patient group is needed.

In conclusion, it is important to investigate zinc deficiency in iron deficient female patients who have memory and attention problems. Zinc supplementation in iron deficient females can have some promising effect on cognitive function.

\section{Conflict of Interest}

The authors of this paper have no conflicts of interest, including specific financial interests, relationships, and/or affiliations relevant to the subject matter or materials included.

\section{References}

1. Aruga, J. 2004. The role of Zic genes in neural development. Mol Cell Neurosci 26(2): 205-221.

2. Bhatnagar, S. and S. Taneja. 2001. Zinc and cognitive development. Br J Nutr 85 Suppl 2: S139-145.

3. Chowanadisai, W., D. M. Graham, C. L. Keen, R. B. Rucker and M. A. Messerli. 2013. A zinc transporter gene required for development of the nervous system. Commun Integr Biol 6(6): e26207.

4. Cumming, T. B., L. Churilov, T. Linden and J. Bernhardt. 2013. Montreal Cognitive Assessment and Mini-Mental State Examination are both valid cognitive tools in stroke. Acta Neurol Scand 128(2): 122-129.

5. Falkingham, M., A. Abdelhamid, P. Curtis, S. Fairweather-Tait, L. Dye and L. Hooper. 2010. The effects of oral iron supplementation on cognition in older children and adults: a systematic review and meta-analysis. Nutr J 9: 4. 
6. Grabrucker, S., T. M. Boeckers and A. M. Grabrucker. 2016. Gender Dependent Evaluation of Autism like Behavior in Mice Exposed to Prenatal Zinc Deficiency. Front Behav Neurosci 10: 37.

7. Hershko, C. and C. Camaschella. 2014. How I treat unexplained refractory iron deficiency anemia. Blood 123(3): 326-333.

8. Jiang, Y. G., H. Y. Fang, W. Pang, J. Liu, H. Lu, Q. Ma and H. T. Fang. 2011. Depressed hippocampal MEK/ERK phosphorylation correlates with impaired cognitive and synaptic function in zinc-deficient rats. Nutr Neurosci 14(2): 45-50.

9. Lozoff, B. and M. K. Georgieff. 2006. Iron deficiency and brain development. Semin Pediatr Neurol 13(3): 158-165.

10. Markiewicz-Zukowska, R., A. Gutowska and M. H. Borawska. 2015. Serum zinc concentrations correlate with mental and physical status of nursing home residents. PLoS One 10(1): e0117257.

11. Maylor, E. A., E. E. Simpson, D. L. Secker, N. Meunier, M. Andriollo-Sanchez, A. Polito, B. Stewart-Knox, C. McConville, J. M. O'Connor and C. Coudray. 2006. Effects of zinc supplementation on cognitive function in healthy middle-aged and older adults: the ZENITH study. Br J Nutr 96(4): 752-760.

12. Nasreddine, Z. S., N. A. Phillips, V. Bedirian, S. Charbonneau, V. Whitehead, I. Collin, J. L. Cummings and H. Chertkow. 2005. The Montreal Cognitive Assessment, MoCA: a brief screening tool for mild cognitive impairment. J Am Geriatr Soc 53(4): 695699.

13. Petersen, R. C. 2011. Clinical practice. Mild cognitive impairment. N Engl J Med 364(23): 2227-2234.

14. Pfaender, S., K. Fohr, A. K. Lutz, S. Putz, K. Achberger, L. Linta, S. Liebau, T. M. Boeckers and A. M. Grabrucker. 2016. Cellular Zinc Homeostasis Contributes to Neuronal Differentiation in Human Induced Pluripotent Stem Cells. Neural Plast 2016: 3760702 .

15. Selekler, K., B. Cangöz and S. Uluç. 2010. Power of discrimination of Montreal Cognitive Assessment (MOCA) scale in Turkish patients with mild cognitive impairement and Alzheimer's disease. Turk Geriatri Dergisi 13: 166-171.

16. Tan, J. P., L. N. Wang and W. Wang. 2006. A case-control study of risk factors in mild cognitive impairment. Zhonghua Liu Xing Bing Xue Za Zhi 27(1): 55-57. 